\title{
Classification criteria for Sjogren's syndrome: nothing ever stands still!
}

\author{
Simon J Bowman, ${ }^{1}$ Robert I Fox ${ }^{2}$
}

Primary Sjögren's syndrome (SS) probably has the dubious distinction of having more proposed criteria than any other rheumatic condition-certainly this was the case in the 1980s and 1990s (eg, Copenhagen, ${ }^{1}$ Californian, ${ }^{2}$ Greek $^{3}$ and Japanese $^{4}$ criteria). In 2002, Vitali et $a^{5}$ published the revised American European Consensus Group (AECG) criteria, which have now served as the gold standard for over 10 years. In 2012, Shiboski et al published an alternative set of criteria, provisionally adopted by the American College of Rheumatology (ACR). These latter criteria (Sjögren's International Collaborative Clinical Alliance (SICCAACR)) are based on objective criteria and do not necessarily require patients to have symptoms of dry eyes or dry mouth. The study by Rasmussen et $a l^{7}$ examines patients with primary SS in a large cohort who fulfil either the existing AECG criteria and/or the newly proposed SICCAACR criteria, and discusses the similarities and differences between them with some important lessons for the field.

The AECG criteria were developed to screen patients with a specific set of questions regarding dryness and then validate the diagnosis with a series of objective tests that indicated immune factors in the pathogenesis. The questionnaires were developed by an expert consensus panel for sensitivity and specificity as an initial screening tool. The AECG criteria require the presence of four out of six components (one of which is symptomatic dry eyes and a second is symptomatic dry mouth), or three out of the four other objective components, although in either case one of the components has to be positive antibodies and/or a positive biopsy. The four objective components are: (1) positive anti-Ro and/or anti-La antibodies; (2) a positive labial gland biopsy defined as at least one periductal focus of 50 or more lymphocytes per $4 \mathrm{~mm}^{2}$ high powered field; (3) reduced

\footnotetext{
${ }^{1}$ Rheumatology Department, Queen Elizabeth Hospital Birmingham, Birmingham, UK; ${ }^{2}$ Rheumatology Clinic, Scripps Memorial Hospital, La Jolla, California, USA

Correspondence to Professor Simon J Bowman, Queen Elizabeth Hospital Birmingham, Vincent Drive, Edgbaston, Birmingham B15 2TH, UK;

Simon.Bowman@uhb.nhs.uk
}

whole salivary flow of $1 \mathrm{~mL} / \mathrm{min}$ or less (or abnormal scintigraphy/sialographysee below); (4) evidence of reduced tear production on van Bijsterveld staining, or $5 \mathrm{~mm}$ or less of flow in $5 \mathrm{~min}$ on Schirmer blotting paper strips. Only two of these objective components being present the alternative formulation (four out of six components) means in effect that the third objective component can be substituted for by the presence of both dry eyes and dry mouth assessed by defined oral and ocular questions. Secondary SS associated with rheumatoid arthritis or lupus was defined separately.

The SICCA-ACR criteria were developed to help define a uniform group of patients with SS for clinical therapeutic trials.

These alternative criteria require two out of three components-again requiring one to be either a positive biopsy or positive antibodies. As an alternative to anti-Ro/La antibodies, however, a positive antinuclear antibody (ANA) level of 1 in 320 or greater plus a positive rheumatoid factor (RF) is also allowed. The third component is a newly devised ocular staining score (OSS) of 3 or greater. ${ }^{8}$ The analysis in the paper by Shiboski et $a l^{6}$ demonstrates that the AECG criteria and the SICCA-ACR criteria identify virtually identical groups of patients with a high $\kappa$ statistic of overlap of 0.88 . As well as providing initial validation of the SICCAACR criteria these data also paradoxically further validate the AECG criteria in an international dataset that includes North American patients. It is perhaps not surprising that there is a great deal of overlap because patient recruitment to the SICCA study included patient support groups (who would probably have fulfilled AECG criteria) as well as patients from other physicians at the tertiary centres involved in the consortia. The ACR validation was thus provisional on the basis of validation in an independent cohort.

The paper by Rasmussen et $a l^{7}$ sheds further light on this issue. In a large wellcharacterised, independent, cohort of 646 patients from the USA it provides independent validation of both the SICCAACR and AECG criteria, again with a high $\kappa$ statistic of 0.81 . Rasmussen $e t a l^{7}$ also examined the genetic underpinning of the two criteria in a subgroup of 180 patients and identified no statistical difference between the choice of criteria in distinguishing patients from controls using this 'biological' measure.

The critical patients in the Rasmussen study (as in the Shiboski study) are those that fulfil one criteria but not the other. Although this is a small group, it is important to ask how they might alter the inclusion or outcome results for a clinical trial. In the case of the 35 individuals who were AECG positive SICCA-ACR negative, they had a positive Schirmer test of ocular dryness or reduced unstimulated salivary flow rate but a negative OSS. Of the 24 patients who were AECG negative SICCA-ACR positive, seven had a positive ANA plus RF only, 17 had a positive OSS of 3 or greater and had either positive histology or serology but without fulfilling other objective AECG criteria. In directly comparing the van Bijsterveld and OSS scoring systems, no patients were positive on the van Bijsterveld staining score but negative using the OSS whereas the converse was not the case. The OSS was found to be more sensitive but less specific.

Rasmussen et $a l^{7}$ propose that those patients who fulfil the AECG criteria but not the SICCA-ACR criteria should be considered to have SS while those patients who fulfil the SICCA-ACR criteria only would generally not be so considered; that is, fundamentally they support the AECG criteria in preference to the SICCA-ACR criteria. They make the point that the AECG criteria can be applied to many patients to confirm the classification in a rheumatologist's office without biopsy or slit lamp examination and there may be circumstances where this is helpful. Nevertheless, they also recognise the importance of these evaluations and that the SICCA-ACR criteria may have advantages in other circumstances.

It is clear that symptoms are not useful in distinguishing patients with SS from those without. Nevertheless, the entry criteria into the SICCA cohort included the requirement for the presence of oral and/ or ocular symptoms or a suspicion of SS; $96 \%$ of patients (ie, nearly all of them in practice) had at least one dryness symptom. In the cohort of Rasmussen et $a l^{7}$ all the participants had to have both oral and ocular dryness symptoms to be recruited into the clinic. In other words, although symptoms are not sufficient to distinguish patients with or without SS, they are inherently part of the SICCA-ACR criteria by virtue of the entry requirements for the SICCA cohort. 
It is arguable, therefore, whether either criteria have been fully validated for asymptomatic patients. Furthermore, both the papers by Shiboski et $a l^{6}$ and by Rasmussen $\mathrm{et}_{\mathrm{al}} \mathrm{l}^{\mathrm{v}}$ validate the use of the combinations of symptoms, whole salivary flow rate and the Schirmer test, as per the AECG formulation of the criteria even though individually these items have low specificity for SS. In a similar vein, the number of patients with secondary SS $(n=87,6 \%)$ in the SICCA cohort was too low to validate the criteria in such patients; that is, the scientific validation of the SICCA-ACR criteria applies to patients with primary SS only.

So where does this leave the Sjogren's research community? Essentially we now have two scientifically validated criteria. The AECG criteria have been used for over 10 years and include over 1300 published papers including clinical trials. They have been the basis for developing both disease activity and disease damage scores. They are not perfect and the data from Shiboski et $a l^{6}$ and Rasmussen et $a l^{7}$ may provide the opportunity to introduce revisions that may sensibly update these criteria (see below).

There will be some debate about the status of patients who fulfil only one set of criteria and not the other as set out in the paper by Rasmussen et al. ${ }^{7}$ It is likely that there will be strong proponents of one set of criteria over the other, but with the benefit of the data published by Rasmussen et $a l^{7}$ it is unlikely that other cohort studies will resolve this issue in favour of either set of criteria but rather provide additional data to confirm these existing validation studies.

If the ACR independently adopt the SICCA-ACR criteria without consensus among the Sjogren's research community this may convey a status that the AECG criteria lack, but this will not guarantee widespread take-up given the popularity to date of the AECG criteria and their flexibility and 'usableness' in clinical practice. There is some potential for confusion in the field with two sets of criteria but this will have to be managed; indeed, in this situation, there would be no choice but to do so.

An alternative approach, which has been initiated at the 2012 ACR Annual Meeting in Washington, in a discussion sponsored by the Sjogren's Syndrome Foundation, USA, with representatives of the SICCA consortium and the European League Against Rheumatism (EULAR) Sjogren's working group, is to reformulate both the AECG and SICCA-ACR criteria into a 'blended' criteria covering both sets of components that could be adopted both by ACR and by EULAR. This goal is broadly supported in principle. ${ }^{9}$ There are, for example, some 'quick wins'. It is rare now to use scintigraphy or sialography for clinical diagnosis and omitting these from the AECG criteria would not be a deal breaker.

The ACR criteria have updated the exclusion criteria for SS to exclude patients with the newly defined IgG4 syndrome. ${ }^{10}$ However, the epidemiology of IgG4 patients in regions outside Japan and the overlap in existing SS cohorts remains to be defined. Less than $1 \%$ of patients fulfil the ACR criteria only by virtue of a positive ANA plus RF. While this antibody profile is not unreasonable in clinical practice these patients potentially overlap with other connective tissue diseases, and initial indications are that the SICCA group would exclude these patients from a classification criterion if the consensus in the broader community was to do so. Both criteria have an important similarity, which is that they both utilise the same biopsy features to classify patients. The blended criteria set could find a form of words to reflect its validation in patients with dryness symptoms (as defined by the AECG symptom descriptors) and without another rheumatic disorder. In the paper by Shiboski et $a l^{6}$ it did appear that a positive Schirmer test could substitute for the OSS in terms of specificity if not sensitivity. van Bijsterveld staining may potentially have similar characteristics. Salivary flow is an interesting omission from the SICCAACR criteria. Salivary gland ultrasound is not currently included in either set of criteria but is increasingly being used in clinical practice and may be an important component of SS criteria in the future, most likely replacing scintigraphy/sialography (which are now rarely performed for diagnostic purposes), although the use of ultrasound in classification criteria will require standardisation and validation before it can be considered a reliable tool for diagnosis and classification in SS. ${ }^{11} 12$

In summary, therefore, our view is that following the publication of the paper by Rasmussen et $a l^{7}$ we now have sufficient data to refine the AECG and provisional SICCA-ACR criteria into a single consensus formulation, albeit with different options for classification and with the role of ultrasound yet to be defined in future studies. There would then be a compelling argument for adoption by the ACR and EULAR. Not to do so would be a missed opportunity that might not come again for some time.

\section{Competing interests None.}

Provenance and peer review Not commissioned; internally peer reviewed.

To cite Bowman SJ, Fox RI. Ann Rheum Dis 2014;73:1-2.

Received 9 July 2013

Revised 12 August 2013

Accepted 19 September 2013

Published Online First 3 October 2013

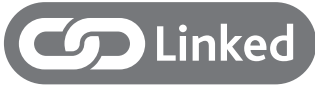

http://dx.doi.org/10.1136/annrheumdis-2013203845

Ann Rheum Dis 2014;73:1-2.

doi:10.1136/annrheumdis-2013-203953

\section{REFERENCES}

1 Manthorpe R, Oxholm P, Prause JU, et al. The Copenhagen criteria for Sjogren's syndrome. Scand J Rheumatol 1986;(Suppl 61):19-21.

2 Fox RI, Robinson CA, Curd JG, et al. Sjogren's syndrome. Proposed criteria for classification. Arthritis Rheum 1986;29:577-85.

3 Skopouli FN, Drosos AA, Papaioannou T, et al. Preliminary diagnostic criteria for Sjogren's syndrome. Scand J Rheumatol 1986;(Suppl 61):22-5.

4 Homma M, Tojo T, Akizuki M, et al. Criteria for Sjogren's syndrome in Japan. Scand J Rheumatol 1986;(Suppl 61):26-7.

5 Vitali C, Bombardieri S, Jonsson R, et al. Classification criteria for Sjogren's syndrome: a revised version of the European criteria proposed by the American European Consensus Group. Ann Rheum Dis 2002;61:554-8.

6 Shiboski S, Shiboski CH, Criswell L, et al. American College of Rheumatology classification criteria for Sjogren's syndrome: a data-driven, expert consensus approach in the SICCA cohort. Arthritis Care \& Research 2012:64:475-87.

7 Rasmussen A, Ice J, Li H, et al. Comparison of the American-European Consensus Group Sjögren's syndrome classification criteria to newly proposed American College of Rheumatology criteria in a large, carefully characterized SICCA cohort. Ann Rheum Dis 2014:73:31-8.

8 Whitcher JP, Shiboski CH, Shiboski SC, et al. A simplified quantitative method for assessing keratoconjunctivitis SICCA from the Sjögren's Syndrome International Registry. Am J Ophthalmol 2010;149:405-15.

9 Vitali C, Bootsma H, Bowman SJ, et al. Classification criteria for Sjogren's syndrome. We actually need to definitively resolve the long debate on the issue. Ann Rheum Dis 2013;72:476-8.

10 Masaki Y, Dong L, Kurose N, et al. Proposal for a new clinical entity, IgG4-positive multiorgan lymphoproliferative syndrome: analysis of 64 cases of IgG4-related disorders. Ann Rheum Dis 2009:68:1310-15.

11 Cornec, et al Contribution of salivary gland ultrasonography to the diagnosis of Sjogren's syndrome. Towards new diagnostic criteria? Arthritis Rheum 2013:65:216-25.

12 Salaffi $F$, Carotti M, lagnocco A, et al. Ultrasonography of salivary glands in primary Sjogren's syndrome: a comparison with contrast sialography and scintigraphy. Rheumatology (Oxford) 2008:47:1244-9. 